\title{
Diversidad de peces en la Reserva de la Biosfera Pantanos de Centla
}

\section{Fish diversity in the Reserva de la Biosfera Pantanos de Centla}

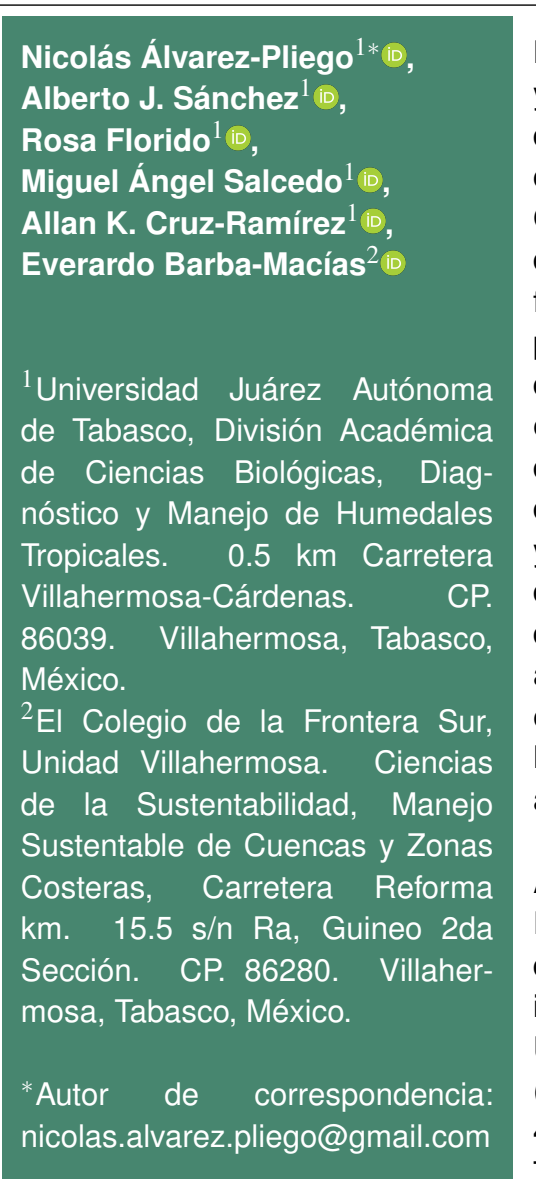

Artículo científico

Recibido: 31 de agosto 2020

Aceptado: 12 de julio 2021

Como citar: Álvarez-Pliego N, Sánchez AJ, Florido R, Salcedo MA, Cruz-Ramírez AK, BarbaMacías E (2021) Diversidad de peces en la Reserva de la Biosfera Pantanos de Centla. Ecosistemas y Recursos Agropecuarios Núm. Esp. I: e2713. DOI: 10.19136/era.a8nl.2713
RESUMEN. Se presenta una lista sistemática de peces recolectados durante 2014 y 2015 en la Reserva de la Biosfera Pantanos de Centla con un incremento del 25\% de su diversidad principalmente por la presencia de peces diádromos. El inventario comprende 75 especies, que representa el $37 \%$ de la ictiofauna en la cuenca Grijalva-Usumacinta, de las cuales cinco especies fueron no nativas invasoras donde el bagre armado (Pterygoplichthys spp.) fue el que presentó la mayor frecuencia de captura. Se identificaron 48 especies migratorias (38 diádromas y 10 potamodromas). La dominancia de peces diádromos y su presencia en sistemas de lagunas y fluviales nos indica el mantenimiento de la interconexión hidráulica entre ríos y lagunas. En los muestreos, se observó la ausencia de siete especies dulceacuícolas que fueron comunes en publicaciones previas, lo anterior puede ser debido a la disminución de hábitats como consecuencia de las actividades humanas y la introducción de especies invasoras. En este estudio se resalta la necesidad de mayores estudios enfocados a la biología de varias especies, con el objetivo de aclarar su condición de potamodromia y comprender la migración lateral que algunas especies presentan durante su ontogenia para satisfacer sus necesidades eco-fisiológicas.

Palabras clave: Ictiofauna, peces migratorios, Grijalva-Usumacinta, humedales, áreas naturales protegidas.

ABSTRACT. The systematic list of fish collected during 2014 and 2015 in the Pantanos de Centla Biosphere Reserve registered a $25 \%$ increase in the previous diversity records, mainly due to the presence of diadromous fish. The checklist included 75 species, accounting for $37 \%$ of the ichthyofauna in the GrijalvaUsumacinta basin. Among the five invasive non-native species, the armored catfish (Pterygoplichthys spp.) registered the maximum frequency of capture. Moreover, 48 migratory species were identified (38 diadromous and 10 potamodromous). The dominance of diadromous fish and their presence in both lagoons and rivers indicated the conservation of the hydraulic interconnection between rivers and lagoons. In the sampling, the absence of seven freshwater species, that were common in previous publications was observed. The above may be due to the decrease in habitats as a consequence of human activities and the introduction of invasive species. This study highlights the need for further studies focused on the fish biology, mainly to clarify the potamodromous condition and understand the lateral migration that some species present during their ontogeny.

Key words: Ichthyofauna, Migratory fish, Grijalva-Usumacinta, wetlands, protected natural areas. 


\section{INTRODUCCIÓN}

La Reserva de la Biosfera Pantanos de Centla (RBPC) está ubicada en la planicie de inundación de las cuencas Grijalva-Usumacinta en el sureste de México, y su hidrografía incluye: cuatro ríos interconectados con aproximadamente 110 lagunas por medio de arroyos, drenes y canales artificiales, que sumadas a las áreas de inundación temporal ocupan más del $48 \%$ del área del polígono (Barba-Macías et al. 2015). Tres de estos ríos drenan permanentemente en el sur del Golfo de México. Los hábitats acuáticos están representados por sustratos suaves sin vegetación, asociaciones de macrófitas acuáticas y arbóreas (Macossay-Cortez et al. 2011, Sánchez et al. 2012b). Todo lo anterior, propicia condiciones para el reclutamiento y establecimiento de peces marinos, estuarinos y dulceacuícolas (Benitez et al. 2018) cuyos patrones de distribución se relacionan con los atributos del hábitat y la disponibilidad de alimento y la ontogenia de los peces (Lucas y Baras 2001).

En la RBPC, los peces son un grupo faunístico acuático dominante (Mendoza Carranza et al. 2010, Macossay-Cortez et al. 2011, Sánchez et al. 2012a), ya que el número de especies en esta área natural comprende a casi el $24 \%$ de los peces nativos reportados para las cuencas de los ríos GrijalvaUsumacinta en territorio mexicano (Soria-Barreto et al. 2018, Sánchez et al. 2019) y su pesca representa casi una quinta parte de la producción pesquera del estado de Tabasco (INE 2000). En los últimos 10 años se ha generado poca información sobre la diversidad de peces distribuidos en la Reserva, en los que se incluyen registros de especies no nativas invasoras (Sánchez et al. 2015b); la ictiofauna en localidades selectas de la RBPC (Soria-Barreto et al. 2018); el reporte puntual de una especie de condrictio no registrado antes en la zona (ReyesRamírez et al. 2018) y el análisis de la diversidad trófica de una comunidad de cíclidos en una laguna de la Reserva (Pease et al. 2018). Pero la diversidad actual de peces en la RBPC aún registra limitaciones de muestreo, actualización en su sistemática y de e- fectos de las especies migratorias marinas. Con respecto a la carencia de muestreos específicos dirigidos a peces de talla pequeña (menos de $50 \mathrm{~mm}$ de longitud patrón), esta condición es una limitante para estimar la totalidad de la diversidad íctica, ya que son peces diversos con distribución asociada a hábitats con estructuras emergentes del sustrato (Olden et al. 2007, Álvarez-Pliego et al. 2016). Los diversos cambios en la sistemática de los peces surgidos en los últimos 15 años, que incluyen desde la modificación de los nombres científicos publicados (Schmitter-Soto 2007, McMahan et al. 2011, Morgenstern 2018) hasta las propuestas de nuevos órdenes (Betancur-R. et al. 2013), aún no han sido consideradas totalmente en las especies de peces distribuidos en la RBPC y en general en la cuenca Grijalva-Usumacinta. Por ejemplo, hay publicaciones que mantienen imprecisiones por desactualización (Castillo-Torres et al. 2017, Calzada-Ruíz et al. 2019, Toledo-Solis et al. 2020). Finalmente, la dinámica de la comunidad íctica de afinidad marina o dulceacuícola que migran o se mueven al interior de la Reserva para cubrir sus requerimientos biológicos es poco conocida, aunque se ha publicado sobre las especies que ingresan durante su etapas iniciales de vida (Arévalo-Frías y Mendoza-Carranza 2012) o para la obtención de recursos (Mendoza-Carranza et al. 2010).

En este contexto, un objetivo fue actualizar el estatus de la riqueza de las especies de peces en la RBPC recolectados del 2014 al 2015 y de su taxonomía. El segundo objetivo incluyó identificar la presencia de las posibles especies migratorias y su frecuencia de ocurrencia en ambientes lóticos y lénticos como reflejo del mantenimiento de la interconexión hidráulica entre ambos tipos de ecosistemas de la RBPC. No obstante, el aumento de las actividades antropogénicas y de la población residente en la Reserva (Barba-Macias et al. 2015, De la Rosa-Velázquez et al. 2017) se espera un incremento de la ictiodiversidad que puede incluir especies invasoras no nativas adicionales. 


\section{MATERIALES Y MÉTODOS}

\section{Descripción del área de estudio}

La RBPC se ubicada en la provincia biótica del Usumacinta (Minckley et al. 2005), en la planicie costera del sur del Golfo de México entre las coordenadas: $17^{\circ} 57^{\prime} 53^{\prime \prime}$ - $18^{\circ} 39^{\prime} 03^{\prime \prime} \mathrm{LN}$ y $92^{\circ}$ $06^{\prime} 39^{\prime \prime}$ - $92^{\circ} 47^{\prime} 58^{\prime \prime}$ LO. Es el humedal más extenso de Norteamérica con un área de 302706 ha que equivale a poco más del $13 \%$ de las Áreas Naturales Protegidas de la Región de la Planicie Costera y Golfo de México (CONANP 2018), así como al 1.6\% de la planicie costera del Golfo de México (VelázquezAguirre y Ordaz-Ayala 1992). Los ríos Grijalva y Usumacinta drenan en promedio 115715 millones de $\mathrm{m}^{3}$ de agua al año, a través de la RPBC en el sur del Golfo de México. Por lo que destacan como la segunda mayor descarga fluvial después del sistema fluvial Misisipi-Atchafalaya (Grenz et al. 2017).

Los principales ríos, Grijalva, Usumacinta, Bitzal y San Pedro y San Pablo, junto con los ríos secundarios, Naranjos, Palomillal y canales artificiales para actividad petrolera suman aproximadamente $925 \mathrm{~km}$ de cauce (Puc-Carrasco et al. 2016). De acuerdo con la literatura hay alrededor de 110 lagunas, en la Reserva, con superficie de 13665 ha, las cuales en su mayoría son dulceacuícolas, aunque algunas, como San Pedrito o El Cometa, presentan ambientes oligohalinos (Montalvo-Urgel et al. 2010, Macossay-Cortez et al. 2011). Más del $80 \%$ de las lagunas se encuentran en la porción centro y sur de la reserva (Puc-Carrasco et al. 2016). La vegetación dominante está compuesta por macrófitas acuáticas como Thalia geniculata L., Typha domingensis Pers. así como comunidades arbóreas (Bucida buceras L., Haematoxylum campechianum L., Pachira aquatica Aubl., Rhizophora mangle L.) (Guadarrama y Ortiz 2000).

\section{Riqueza íctica}

La lista se elaboró con base en las recolectas efectuadas durante noviembre de 2014 y marzo, junio, julio y septiembre de 2015. Los peces se capturaron en 39 localidades, 24 en ecosistemas lénticos y 15 en lóticos (Tabla 1). Las artes de pesca utilizadas fueron: red de cerco de playa de $3 \mathrm{~m}$ de largo, $2 \mathrm{~m}$ de caída y $1 \mathrm{~cm}$ de malla (10 repeticiones por sitio); una red tipo atarraya con $1 \mathrm{~cm}$ de malla (10 lanzamientos por sitio); una red de enmalle (agallera) de $30 \mathrm{~m}$ de largo, $2 \mathrm{~m}$ de caída y $6.5 \mathrm{~cm}$ de malla (una réplica de 1 hora por sitio); tres redes de cuchara con malla de $1 \mathrm{~mm}$ (tres réplicas de 10 minutos por sitio); trampas tipo nazas (tres por sitio durante 1 hora). Por lo general, las capturas se realizaron en el centro y los márgenes de las lagunas, así como en las orillas de los ríos con poca profundidad o donde se presentaban playones o zonas de baja energía. El número de especies registrado en cada localidad fue anotado y se añadieron prácticas y actividades antropogénicas en cada una de las localidades (Tabla 1).

La identificación taxonómica de las especies nativas y no nativas se corroboró con la literatura científica publicada por Trewavas (1983), CastroAguirre et al. (1999), Miller et al. (2005), Armbruster y Page (2006), Schmitter-Soto (2017) y Sánchez et al. (2019). Los nombres científicos se validaron con el Catálogo de Peces de Eschmeyer (Fricke et al. 2020) y el orden sistemático se estableció utilizando como referencia a Nelson et al. (2016), con excepción de la especie de la familia Polynemidae, ya que no fue incluida en esta última clasificación, en este sentido Polydactylus octonemus (Girard) fue incluida dentro de los Carangiformes de acuerdo a Girard et al. (2020). Los especímenes del bagre armado se identificaron hasta nivel de género (Pterygoplichthys spp.), ya que ante la falta de un estudio genético las características morfológicas son insuficientes para determinar su identidad como lo sugieren Wu et al. (2011), Nico et al. (2012) y Álvarez-Pliego et al. (2015). Finalmente se revisó el estatus de conservación de cada especie de acuerdo a la NOM-059 (SEMARNAT 2010) y a la Lista Roja de Especies Amenazadas de la UICN (2021).

Una vez identificadas las especies, se procedió a definir los criterios para establecer las categorías de especies migratorias, para lo cual se consultaron las propuestas de Myers (1949), Lucas y Baras (2001), Chapman et al. (2012), Schultz y McCormick (2012), y Zydlewski y Wilkie (2012). Con base en esta información se definió como peces 
Tabla 1. Localidades y sitios de recolecta de peces en la Reserva de la Biósfera Pantanos de Centla. Reg, registros por sitio; spp., número de especies registradas.

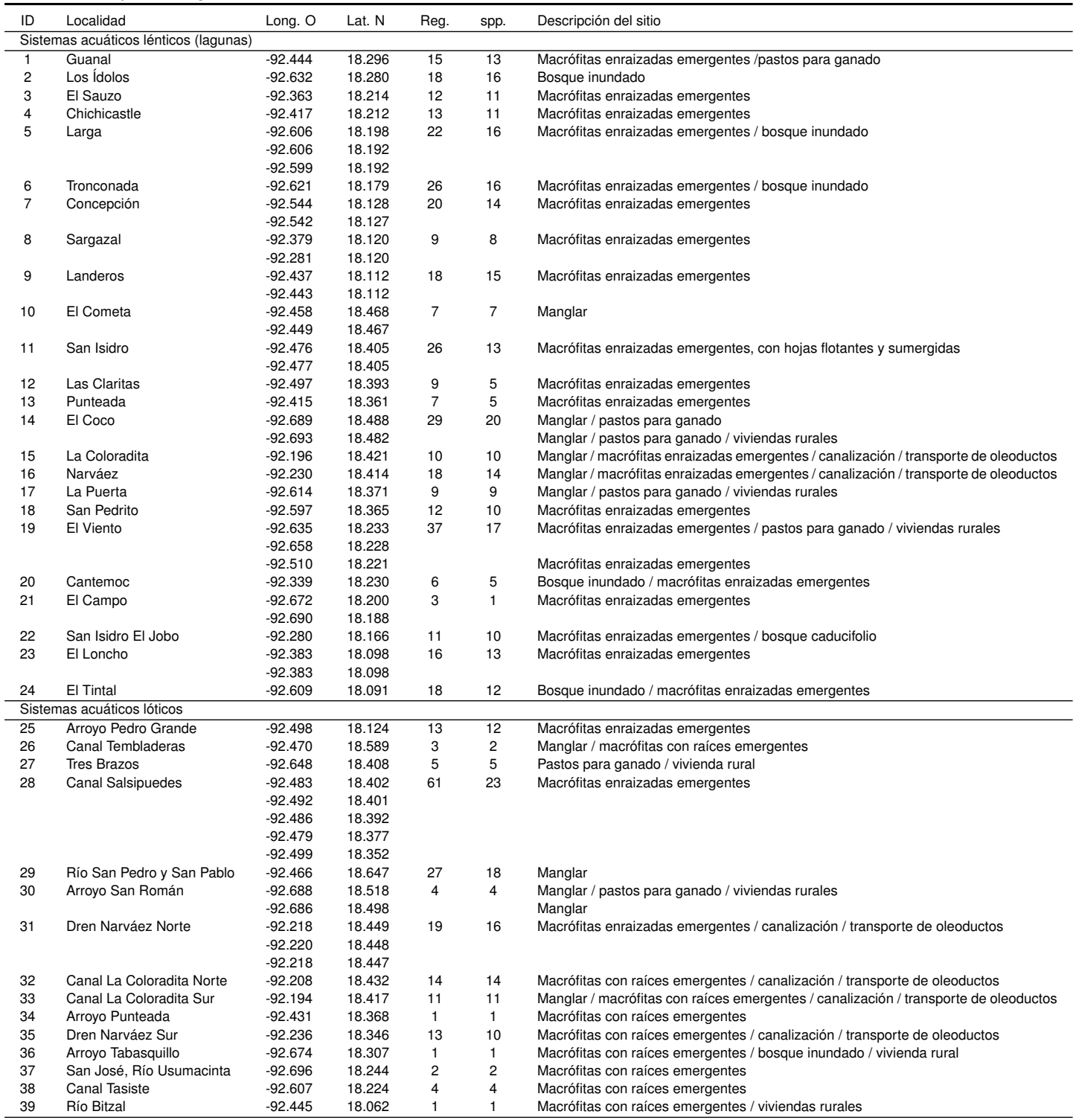

migratorios a aquellos que realizan desplazamientos dados por su ecofisiología. Los peces migratorios se dividieron en especies diádromas y potamodromos. Las primeras son peces que desarrollan mecanismos osmoreguladores para desplazarse por diferentes concentraciones de salinidad. Aquí se incluyen especies anádromas, catádromas, anfídromas así como aquellas eurihalinas que se desplazan desde ecosistemas polihalinos hasta limnéticos. En cambio, las especies potamodromos son peces que migran o se mueven solo entre diferentes hábitats de agua dulce.

En este contexto, se realizó una búsqueda bibliográfica donde se identificaron a las especies como migratorias o con rasgos biológicos suficientes para asignarlas en alguna de las dos categorías. Las referencias revisadas fueron: Castro-Aguirre et al. (1999), Lucas y Baras (2001), Riede (2004), Miller et al. (2005), Milton (2009), Mendoza-Carranza et al. (2010), Adams et al. (2011), McBride y Mathe- 
son (2011), Macossay-Cortez et al. 2011; GalvanQuesada et al. (2016), Soria-Barreto et al. (2018), Benitez et al. (2018), Sánchez et al. (2019) y Froese y Pauly (2021). La cuantificación del número de especies, familias y órdenes resultantes de este estudio fue comparada con la composición histórica de la riqueza ictiofaunística ya registrada en ReséndezMedina y Salvadores (2000), Mendoza-Carranza et al. (2010) y Macossay-Cortez et al. (2011); y se comparó la distribución de especies capturadas en los ambientes lacustres y fluviales. Fue contrastada con los datos obtenidos por Macossay-Cortez et al. (2011), con énfasis en la presencia de especies migratorias.

El material aquí reportado, se encuentra depositado en la Colección Nacional de Peces del Instituto de Biología de la UNAM (CNP-IBUNAM 21235 al CNP-IBUNAM 21412; CNP-IBUNAM 21401 al CNP-IBUNAM 21412; CNP-IBUNAM 21583 al CNP-IBUNAM 21764; CNP-IBUNAM 21836 al CNPIBUNAM 21886; CNP-IBUNAM 21985 al CNPIBUNAM 22115).

\section{RESULTADOS}

El elenco se compuso por dos clases, 19 órdenes, 28 familias, 64 géneros y 75 especies. Veintiún especies se adicionaron a la ictiofauna de la RBPC, de las cuales 17 son de afinidad marina (Tabla 2). Las familias mejor representadas por su número de especies fueron Cichlidae con 12 especies (10 autóctonas), y Poeciliidae con nueves especies. Cinco especies no nativas invasoras fueron identificadas: Ctenopharyngodon idella (Valenciennes), Cyprinus carpio Linnaeus, Pterygoplichthys spp., Oreochromis niloticus (Linnaeus) y Parachromis motaguensis (Günther). El bagre armado Pterygoplichthys spp. estuvo presente en 27 de las 39 localidades. También se determinaron 48 especies de peces clasificados como migratorios, entre ellos 38 diádromos y 10 potamodromos (Tabla 2). Potamarius nelsoni (Evermann y Goldsborough) y Rhamdia guatemalensis (Günther) fueron las únicas especies (de la presente lista) incluidas en la NOM-059 como sujetas a protección especial. En la Lista Roja de Especies Amenazadas de la UICN, Ctenogobius claytonii (Meek) y Megalops atlanticus Valenciennes aparecen con el estatus de vulnerables. En esta misma lista también son mencionadas 34 especies más, pero con las categorías de preocupación menor (26) y datos insuficientes (8) para valorar su situación.

En relación a la riqueza histórica de la ictiofauna de la RBPC, comprende $86 \mathrm{spp}$. agrupadas en 31 familias y 20 órdenes (Tabla 3). Los órdenes mejor representados numéricamente y más frecuentes fueron los Cichliformes, Cyprinodontiformes y Perciformes. Los grupos que registraron algún incremento en su diversidad fueron los Clupeiformes, Gobiiformes y Siluriformes, mientras que especies de los Syngnathiformes no fueron capturadas. El elevado número de familias de peces obtenido en este estudio (28) se explica por el incremento de especies marinas por el cual se adicionaron algunas como Carangidae, Dasyatidae y Polynemidae a la ictiofauna de la RBPC (Tabla 3).

El número de especies recolectadas por tipo de ambiente resultó mayor en los lénticos que en los lóticos. En las lagunas se registraron 58 especies (incluidas 33 migratorias), de las cuales 24 fueron obtenidas exclusivamente en estos ambientes. Las especies con mayor frecuencia de captura, más del $50 \%$ de sitios lénticos, fueron: Pterygoplichthys spp., Thorichthys helleri (Steindachner), Thorichthys pasionis (Rivas), Cathorops aguadulce (Meek), Astyanax finitimus (Bocourt), Thorichthys meeki Brind, Dorosoma petenense (Günther), Trichromis salvini (Günther) y Vieja melanurus (Günther). En el caso del bagre armado sus ejemplares fueron capturados en el $90 \%$ de los sitios en lagunas.

En los ecosistemas fluviales su riqueza fue de 51 especies, de las cuales 17 fueron capturadas sólo en estos ambientes lóticos. Las especies Trichromis salvini, Gambusia sexradiata Hubbs, Gambusia yucatana Regan, Phallichthys fairweatheri Rosen \& Bailey y Pterygoplichthys spp. se consideraron frecuentes, ya que se capturaron en más del $40 \%$ de las localidades. Del total de especies capturadas en estos ambientes, 30 fueron reconocidas como migratorias (Tabla 4). Sólo el $28 \%$ de las especies migrato- 
Tabla 2. Lista sistemática de los peces recolectados por localidad en la RBPC. * Especies diádromas, ** especies potamodromas, i especies no nativas invasoras, $\dagger$ nuevos registros para la Reserva. $€$ especies mencionadas en la NOM-059 y en la lista de la UICN. El número corresponde al ID de las localidades de muestreo, se pueden consultar en la Tabla 1.

\begin{tabular}{|c|c|c|}
\hline Orden / Familia / Especie & Localidades lénticas (ID) & Localidades lóticas (ID) \\
\hline \multicolumn{3}{|l|}{ Myliobatiformes / Dasyatidae } \\
\hline \multicolumn{3}{|l|}{ Lepisosteiformes / Lepisosteidae } \\
\hline Atractosteus tropicus Gill $1863 €$ & 7,10 & 32,33 \\
\hline Elops saurus Linnaeus 1766 * & & 29 \\
\hline \multicolumn{3}{|l|}{ Elopiformes / Megalopidae } \\
\hline \multicolumn{3}{|l|}{ Clupeiformes / Engraulidae } \\
\hline Brevoortia gunteri Hildebrand $1948^{*} \dagger$ & 14 & \\
\hline Dorosoma anale Meek $1904^{\star \star} €$ & $1,2,5,6,7,8,9,19,20,24$ & $25,28,31$ \\
\hline Dorosoma petenense (Günther 1867 ) ${ }^{\star} €$ & $1,3,4,5,6,7,8,9,19,20,23,24$ & 29,31 \\
\hline Harengula jaguana Poey $1865 * \dagger$ & & 29 \\
\hline \multicolumn{3}{|l|}{ Cypriniformes / Cyprinidae } \\
\hline Cyprinus carpio Linnaeus $1758 \mathrm{i}^{\star \star}$ & 16 & \\
\hline Ctenopharyngodon idella (Valenciennes 1844) ${ }^{* *} \mathrm{i}$ & 35 & \\
\hline \multicolumn{3}{|c|}{ 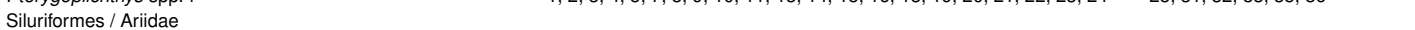 } \\
\hline Ariopsis felis (Linnaeus 1766) * & 26,29 & \\
\hline Bagre marinus (Mitchill 1815) * & 14 & \\
\hline Cathorops aguadulce (Meek 1904) * & $1,2,3,4,8,9,10,14,17,19,20,22,23,24$ & $28,29,30,38$ \\
\hline \multicolumn{3}{|l|}{ Siluriformes / Heptapteridae } \\
\hline Rhamdia guatemalensis (Günther 1864) $€$ & 5 & \\
\hline Rhamdia laticauda (Kner 1858) $\dagger$ & 14 & 28,29 \\
\hline Batrachoidiformes / Batrachoididae & & \\
\hline Opsanus beta (Goode y Bean 1880) * $† €$ & 17 & \\
\hline Gobiiformes / Eleotridae & & \\
\hline Dormitator maculatus (Bloch 1792) * $€$ & 2 & 28,34 \\
\hline Eleotris amblyopsis (Cope 1871) * $€$ & 2,14 & 28,31 \\
\hline Gobiomorus dormitor Lacepède $1800^{*} €$ & 9,18 & 28,32 \\
\hline Oscura heterospila (Hubbs 1936) $€$ & $4,5,6,7,9,18,19$ & \\
\hline Parachromis motaguensis (Günther 1867) i & 5 & \\
\hline Parachromis multifasciatus (Regan 1905) & $11,14,24$ & \\
\hline Petenia splendida Günther $1862 €$ & $1,4,8,10,16,19,23$ & $25,28,32,35$ \\
\hline Thorichthys helleri (Steindachner 1864) $€$ & $1,2,3,4,5,6,7,9,11,13,14,16,17,19,22,23,24$ & $25,35,38$ \\
\hline Thorichthys meeki Brind 1918 & $1,2,4,5,6,9,11,13,18,19,22,23,24$ & \\
\hline Thorichthys pasionis (Rivas 1962) & $1,2,3,5,6,7,8,9,11,13,15,16,19,20,22,23$ & $25,28,32$ \\
\hline Trichromis salvini (Günther 1862) $€$ & $2,5,6,7,11,12,15,16,18,19,22,23$ & $25,27,28,30,31,32,33,35,38$ \\
\hline Vieja melanurus (Günther 1862) $€$ & $2,3,4,5,6,7,9,15,16,18,23,24$ & $25,28,31,32,35$ \\
\hline Atheriniformes / Atherinopsidae & & \\
\hline Atherinella alvarezi (Díaz-Pardo 1972) $€$ & 1 & \\
\hline Membras martinica (Valenciennes 1835 ) * $\dagger €$ & & 29 \\
\hline Beloniformes / Hemiramphidae & & \\
\hline Hyporhamphus mexicanus Álvarez $1959^{* \star} €$ & 6,10 & \\
\hline Beloniformes / Belonidae & & \\
\hline Strongylura hubbsi Collette $1974^{\star \star} \dagger €$ & $1,3,4,19$ & \\
\hline Cyprinodontiformes / Poeciliidae & & \\
\hline Belonesox belizanus Kner $1860 €$ & 31,32 & \\
\hline Carlhubbsia kidderi (Hubbs 1936) $€$ & 6 & 28,33 \\
\hline Gambusia sexradiata Hubbs $1936 €$ & $6,8,9,11,12,15,19,23,24$ & $25,27,28,31,32,33,35$ \\
\hline Gambusia yucatana Regan $1914 €$ & $9,11,15,16$ & $27,28,31,32,33,35$ \\
\hline Heterophallus echeagarayi (Álvarez 1952) $€$ & $3,14,16,19$ & $27,28,30,31,37$ \\
\hline Phallichthys fairweatheri Rosen y Bailey $1959 €$ & $6,11,12,15,19,22,24$ & $25,28,31,32,33,35$ \\
\hline Poecilia mexicana Steindachner $1863 €$ & $14,15,24$ & $27,28,31$ \\
\hline Pseudoxiphophorus bimaculatus (Heckel 1848) $€$ & $28,32,33$ & \\
\hline Xiphophorus maculatus (Günther 1866) $€$ & 11,12 & 28 \\
\hline Synbranchiformes / Synbranchidae & & \\
\hline Ophisternon aenigmaticum Rosen y Greenwood 1976 & 15,22 & 28,33 \\
\hline
\end{tabular}




\begin{tabular}{|c|c|c|}
\hline \multirow{2}{*}{\multicolumn{3}{|c|}{$\begin{array}{l}\text { Orden / Familia / Especie } \\
\text { Carangiformes / Carangidae }\end{array}$}} \\
\hline & & \\
\hline \multicolumn{2}{|l|}{ Caranx hippos (Linnaeus 1766) * $\dagger €$} & 29 \\
\hline \multicolumn{2}{|l|}{ Caranx latus Agassiz $1831^{*} \dagger €$} & 29 \\
\hline \multicolumn{2}{|l|}{ Oligoplites saurus (Bloch y Schneider 1801) * $\dagger €$} & 29 \\
\hline \multicolumn{2}{|l|}{ Selene setapinnis (Mitchill 1815) * $\dagger$} & 29 \\
\hline \multicolumn{2}{|l|}{ Trachinotus carolinus (Linnaeus 1766) * $\dagger$} & 29 \\
\hline \multicolumn{3}{|l|}{ Carangiformes / Polynemidae } \\
\hline \multicolumn{2}{|l|}{ Polydactylus octonemus (Girard 1858$)^{*} \dagger$} & 29 \\
\hline \multicolumn{3}{|l|}{ Pleuronectiformes / Paralichthyidae } \\
\hline \multicolumn{3}{|l|}{ Citharichthys spilopterus Günther 1862 * } \\
\hline \multicolumn{3}{|l|}{ Pleuronectiformes / Achiridae } \\
\hline Achirus lineatus (Linnaeus 1758) * & 14 & \\
\hline \multicolumn{3}{|l|}{$\begin{array}{l}\text { Trinectes maculatus (Bloch y Schneider 1801) * } \\
\text { Perciformes / Centropomidae }\end{array}$} \\
\hline \multicolumn{3}{|l|}{ Centropomus mexicanus Bocourt $1868^{*} \dagger €$} \\
\hline \multirow{2}{*}{\multicolumn{3}{|c|}{$\begin{array}{l}\text { Centropomus parallelus Poey } 1860 \text { * } € \\
\text { Perciformes / Gerreidae }\end{array}$}} \\
\hline & & \\
\hline \multicolumn{3}{|l|}{ Diapterus auratus Ranzani 1842 * } \\
\hline Diapterus rhombeus (Cuvier 1829) * $\dagger$ & 14 & 30 \\
\hline \multicolumn{2}{|l|}{ Eucinostomus melanopterus (Bleeker 1863) * $\dagger$} & 29 \\
\hline Eugerres mexicanus (Steindachner 1863) $\dagger$ & $5,6,7,13,16$ & 25 \\
\hline \multirow{2}{*}{\multicolumn{3}{|c|}{$\begin{array}{l}\text { Eugerres plumieri (Cuvier 1830) }{ }^{*} \\
\text { Acanthuriformes / Sciaenidae }\end{array}$}} \\
\hline & & \\
\hline \multicolumn{3}{|l|}{ Aplodinotus grunniens Rafinesque 1819} \\
\hline Bairdiella chrysoura (Lacepède 1802) * & 14,17 & \\
\hline Bairdiella ronchus (Cuvier 1830) * & 2 & \\
\hline Cynoscion arenarius Ginsburg 1930 * & 14 & \\
\hline Micropogonias undulatus (Linnaeus 1766) * $\dagger$ & 18 & \\
\hline Número de especies por tipo de ambiente & 58 & 51 \\
\hline Especies registradas en un solo tipo de ambiente & 24 & 17 \\
\hline Número de especies registrada en ambos ambientes & 34 & \\
\hline
\end{tabular}

Tabla 3. Comparación de la ictiofauna en la RBPC registrada en publicaciones previas y en el presente estudio. Las familias señaladas en negritas son las nuevas adiciones de cada referencia. Se incluye el número de las especies (spp.) y los órdenes registrados.

\begin{tabular}{|c|c|c|c|c|}
\hline Publicación & Ictiofauna & spp. & Familias presentes & Órdenes \\
\hline $\begin{array}{l}\text { Reséndez-Medina y } \\
\text { Salvadores (2000) }\end{array}$ & $\begin{array}{l}\text { Nativas } \\
\text { No nativas invasoras } \\
\text { Diádromas } \\
\text { Potamodromas }\end{array}$ & $\begin{array}{c}22 \\
3 \\
11 \\
3\end{array}$ & $\begin{array}{l}\text { Ariidae, Centropomidae, Cichlidae, Clupeidae, Cyprinidae, Eleotridae, } \\
\text { Elopidae, Gerreidae, Heptapteridae, Lepisosteidae, Lutjanidae, } \\
\text { Megalopidae, Mugilidae, Poeciliidae, Sciaenidae. }\end{array}$ & 11 \\
\hline $\begin{array}{l}\text { Mendoza-Carranza } \\
\text { et al. (2010) }\end{array}$ & $\begin{array}{l}\text { Nativas } \\
\text { No nativas invasoras } \\
\text { Diádromas } \\
\text { Potamodromas }\end{array}$ & $\begin{array}{c}25 \\
4 \\
10 \\
3\end{array}$ & $\begin{array}{l}\text { Achiridae, Atherinopsidae, Batrachoididae, Belonidae, Cichlidae, } \\
\text { Characidae, Clupeidae, Cyprinidae, Eleotridae, Engraulidae, Gerreidae, } \\
\text { Heptapteridae, Lepisosteidae, Loricariidae, Mugilidae, Syngnathidae. }\end{array}$ & 14 \\
\hline $\begin{array}{l}\text { Macossay-Cortez et } \\
\text { al. (2011) }\end{array}$ & $\begin{array}{l}\text { Nativas } \\
\text { No nativas invasoras } \\
\text { Diádromas } \\
\text { Potamodromas }\end{array}$ & $\begin{array}{c}43 \\
1 \\
13 \\
5\end{array}$ & $\begin{array}{l}\text { Achiridae, Ariidae, Atherinopsidae, Batrachoididae, Belonidae, Cichlidae, } \\
\text { Characidae, Clupeidae, Eleotridae, Engraulidae, Gerreidae, Gobiidae, } \\
\text { Heptapteridae, Hemiramphidae, Lepisosteidae, Loricariidae, Mugilidae, } \\
\text { Paralichthyidae, Poeciliidae, Rivulidae, Sciaenidae, Synbranchidae, }\end{array}$ & 13 \\
\hline Presente trabajo & $\begin{array}{l}\text { Nativas } \\
\text { No nativas invasoras } \\
\text { Diádromas } \\
\text { Potamodromas }\end{array}$ & $\begin{array}{c}70 \\
5 \\
38 \\
10\end{array}$ & $\begin{array}{l}\text { Achiridae, Ariidae, Atherinopsidae, Batrachoididae, Belonidae, Carangidae, } \\
\text { Centropomidae, Characidae, Cichlidae, Clupeidae, Cyprinidae, Dasyatidae, } \\
\text { Eleotridae, Elopidae, Engraulidae, Gerreidae, Gobiidae, Hemiramphidae, } \\
\text { Heptapteridae, Lepisosteidae, Loricariidae, Megalopidae, Mugilidae, } \\
\text { Paralichthyidae, Poeciliidae, Polynemidae, Sciaenidae, Synbranchidae. }\end{array}$ & 19 \\
\hline
\end{tabular}

rias reportadas en este trabajo fueron obtenidos en ambos ambientes (lóticos y lénticos). Sin embargo, el $66 \%$ de los peces diádromos fueron registrados en las lagunas, siendo las más frecuentes $C$. aguadulce, D. petenense y Diapterus auratus Ranzani. En cambio, Astyanax finitimus (Bocourt), Dorosoma anale Meek, y Hyphessobrycon compressus (Meek) fueron las especies potamodromos de mayor presencia (Tabla 2). La afluencia de especies de afinidad ma- rina a los ambientes fluviales fue muy similar que en lagunas (25 spp.), pero solo $C$. aguadulce estuvo presente en una cuarta parte de estas localidades.

\section{DISCUSIÓN}

La pérdida de la conexión hidráulica entre los ecosistemas lénticos y lóticos es uno de los principales factores asociados con las actividades y prác- 
Tabla 4. Comparación de la ictiofauna de la RBPC asociada a la dinámica de los ecosistemas. Las especies migratorias están señaladas en negritas.

\begin{tabular}{|c|c|c|c|c|c|c|}
\hline \multicolumn{7}{|c|}{ Macossay-Cortez et al. (2011) } \\
\hline & $\begin{array}{l}\text { Diversidad por } \\
\text { ambiente }\end{array}$ & Especies frecuentes & Exclusivas & Diadromos & Potamodromos & Dulceacuícolas \\
\hline 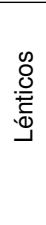 & 19 loc /41 spp. & $\begin{array}{l}\text { A. finitimus, D. maculatus, G. } \\
\text { sexradiata, G. yucatana, G. dor- } \\
\text { mitor, H. compressus, M. uroph- } \\
\text { thalmus, O. aenigmaticum, O. het- } \\
\text { erospila, P. fairweatheri, T. helleri, } \\
\text { T. meeki, T. pasionis, T. salvini, V. } \\
\text { melanurus. }\end{array}$ & 7 spp. & 14 spp. & 3 spp. & $28 \mathrm{spp}$. (todas nativas) \\
\hline \multirow[t]{2}{*}{ 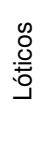 } & 12 loc / 27 spp. & $\begin{array}{l}\text { A. finitimus, D. maculatus, G. } \\
\text { sexradiata, G. yucatana, G. dormi- } \\
\text { tor, H. echeagarayi, P. mexicana. }\end{array}$ & 3 spp. & 5 spp. & 2 spp. & 25 spp. (24 nativas, 1 invasora) \\
\hline & & \multicolumn{5}{|c|}{ Presente estudio } \\
\hline 哭 & 24 loc /58 spp. & $\begin{array}{l}\text { A. finitimus, C. aguadulce, D. } \\
\text { anale, D. petenense, Pterygo- } \\
\text { plichthys spp., T. helleri, T. meeki, } \\
\text { T. pasionis, T. salvini, V. melanurus. }\end{array}$ & $24 \mathrm{spp}$ & 25 spp. & 9 spp. & 32 spp. (29 nativas, 3 invasoras) \\
\hline $\begin{array}{l}0 \\
.0 \\
.0 \\
.0\end{array}$ & 15 loc /51 spp. & $\begin{array}{l}\text { G. sexradiata, G. yucatana, P. fair- } \\
\text { weatheri, Pterygoplichthys spp. }\end{array}$ & 17 spp. & 24 spp. & 6 spp. & 27 spp. (24 nativas, 3 invasoras) \\
\hline
\end{tabular}

ticas antropogénicas que afecta de manera negativa la biodiversidad de los ecosistemas acuáticos (Liu y Wang 2018). Para la planicie de inundación de la cuenca Grijalva-Usumacinta se han reportado impactos negativos en la ictiofauna, los cuales se atribuyen a las actividades humanas, que incluyen la perturbación de la interconexión hidráulica (TorresMartínez et al. 2017, Sánchez et al. 2019). En el caso de Pantanos de Centla se registró una mayor diversidad de peces en las lagunas conectadas que en las aisladas (Cazzanelli et al. 2021). En este sentido, el mantenimiento de la interconexión hidráulica superficial de las áreas naturales, como la de RBPC, es vital para la conservación de los recursos bióticos de una zona considerada de alta biodiversidad (Myers et al. 2000).

La riqueza de especies de peces de la RBPC representa más del $43 \%$ de la diversidad catalogada para la cuenca del río Usumacinta en México (SoriaBarreto et al. 2018) y el $77 \%$ de la riqueza íctica registrada por Gómez-González et al. (2015) y Sánchez et al. (2019) en la cuenca del río Grijalva. El número de especies en Pantanos de Centla es mayor en comparación con otros humedales ubicados en la cuenca del Usumacinta como: Laguna Lachuá (36 spp.) y río San Pedro (33) (Granados-Dieseldorff et al. 2012, Castillo-Domínguez et al. 2015). Aunque, también está asociado a la mayor presencia de especies marinas por su permanente conexión con la zona nerítica del sur del Golfo de México, así como una mayor área.

La riqueza histórica de las especies reportada por Reséndez-Medina y Salvadores (2000), Mendoza-Carranza et al. (2010), Macossay-Cortez et al. (2011) y las incluidas en el presente estudio suman $86 \mathrm{spp}$. (Tabla 3). La menor diversidad (30 especies) fue reportada por Reséndez-Medina y Salvadores (2000), mientras que Mendoza-Carranza et al. (2010) reporta la ausencia del grupo dominante de los Cyprinodontiformes. Esta ausencia se explica por el diseño de muestreo restringido a hábitats selectos (manglares y pastos marinos) y la selectividad de las artes de pesca utilizados que preferentemente capturan especies de tallas mayores a $50 \mathrm{~mm}$ o especímenes adultos, como lo mencionan ÁlvarezPliego et al. (2016).

Se observa una constante incursión de especies marinas provenientes del Golfo de México a Pantanos de Centla. Los peces marinos con capacidad para migrar hacia las lagunas y sistemas fluviales de la Reserva son frecuentes y su proporción histórica ha comprendido entre el 30 y $50 \%$ de la riqueza de la comunidad íctica (Tabla 2 y 3). Esta gran diversidad de peces diádromos 
responde a la alta productividad de recursos y hábitats disponibles que ofrecen este tipo de ecosistemas (Milton 2009). Como es el caso de la RBPC en donde hay disponibilidad de hábitats con estructuras emergentes como macrófitas enraizadas emergentes, flotantes enraizadas, sumergidas enraizadas y libres flotadoras (Macossay-Cortez et al. 2011, Sánchez et al. 2012b). Aunque, la marcada producción de biomasa del fitoplancton puede ser otra fuente de alimento para estos peces en el nivel bajo de inundación (Cruz-Ramírez et al. 2019). En este contexto, algunas de las especies marinas migratorias se capturaron con mayor frecuencia en la presente investigación. Según los criterios publicados por Castro-Aguirre et al. (1999) y Adams et al. (2011) quedan como ejemplos de peces frecuentes anfídromos (C. aguadulce, D. auratus, Dormitator maculatus (Bloch) y Gobiomorus dormitor Lacepède), anádromos ( $M$. lineatus y $D$. petenense) y catádromos (Centropomus mexicanus Bocourt, Centropomus parallelus Poey y Centropomus undecimalis (Bloch)) (Tabla 2).

El bagre $C$. aguadulce se consideró una especie con afinidad a los ambientes estuarinos (ReyesRamírez et al. 2017). No obstante, por sus registros en ambientes limnéticos (Castillo-Domínguez et al. 2015, Sánchez et al. 2019) puede ser considerada como una especie dulceacuícola eurihalina, si se aplica la clasificación de McCormick et al. (2013) o Castro-Aguirre et al. (1999); que sugieren que podría ser una especie en transición a ser vicaria. Otra especie que requiere atención es $D$. petenense ya que McBride y Matheson (2011) consideraron que no es una especie diádroma al menos para las costas de Florida, EUA. Pero por las evidencias sobre su biología (Froese R y Pauly D 2021) se clasificó como una especie migratoria. En el caso de las especies de centropomidos, Castro-Aguirre et al. (1999) sugirieron que C. mexicanus podría tratarse de una forma previcaría ya que su afinidad y presencia en ambientes limnéticos de las cuencas GrijalvaUsumacinta es frecuente.

El $34 \%$ de peces de afinidad marina y estuarina en la RBPC contrasta con el $22 \%$ reportado por Sánchez et al. (2019), en las lagunas urbanas y suburbanas en la planicie costera del río Grijalva, donde fueron recolectadas en lagunas solo ocho especies diádromas (A. mitchilli, B. gunteri, D. maculatus, E. amblyopsis, A. lineatus, y $D$. auratus). Lo anterior se asoció con la fuerte presión de las actividades humanas en las zonas conurbadas (Palomeque et al. 2017). En este aspecto, la urbanización altera la estructura abiótica y biótica de los hábitats acuáticos, por la desconexión de los lagos de sus tributarios en la planicie de inundación (Sánchez et al. 2015b, Zhang et al. 2018). Lo cual impide la migración de los peces y su reemplazo que se asocian con la reducción de su riqueza (Edge et al. 2017).

Así como las especies diádromas, la presencia de peces potamodromos son indicadores de la interconexión entre los ríos y los lagos en la planicie de inundación (Lucas y Baras 2001). No solo los movimientos longitudinales de los peces, también las migraciones laterales hacia la planicie debido a la conexión hidráulica superficial están asociados con un pulso de inundación y la disponibilidad de macrófitas emergentes enraizadas que funcionan como sitios de protección, reproducción y alimentación (Salcedo et al. 2012, Sánchez et al. 2012b, Galván-Quesada et al. 2016).

Astyanax finitimus, $D$. anale y $H$. compressus fueron los peces potamodromos capturados con mayor frecuencia en las lagunas de la Reserva y se sumaron a las especies potamodromos lctiobus meridionalis (Günther) e Ictalurus meridionalis (Günther), las cuales no habían sido mencionadas en publicaciones previas de la RBPC, lo cual puede estar relacionado a la afinidad de estas especies con los ambientes reofilicos (Miller et al. 2005) o a los dispositivos de muestreo utilizados, ya que se ha reportado, que su captura es más efectiva utilizando el arpón y los anzuelos (Mendoza-Carranza et al. 2018).

La preservación y riqueza de la ictiofauna de la RBPC es consecuencia del mantenimiento de la reconexión hidráulica superficial entre lagunas y ríos, ya que la intensidad de la interconexión es un factor regulador de la distribución espacial de la ictiofauna (Liu y Wang 2018, Sánchez et al. 2019). En este sentido, la presencia de especies migratorias, casi 
el $47 \%$ de la ictiofauna, en los ambientes lacustres de la RBPC, es un indicador de la conexión entre el mar, los ríos y las lagunas, dados los flujos bidireccionales y laterales por lo que los organismos de afinidad marino estuarina transitan hacia ambientes limnéticos, se reproducen, se refugian o alimentan y se distribuyen en la planicie (Mendoza-Carranza et al. 2010, Puc-Carrasco et al. 2016, Reyes-Ramírez et al. 2018).

La escasez de registros o la ausencia de siete especies dulceacuícolas en el presente estudio: Brycon guatemalensis Regan, Batrachoides goldmani Evermann y Goldsborough, Cincelichthys pearsei (Hubbs), Parachromis managuensis (Günther), Rocio octofasciata (Regan), Cynodonichthys tenuis Meek, Poecilia kykesis Poeser, abre la hipótesis si la actual disminución de diversidad de especies dulceacuícolas está asociada con la perturbación causada por las actividades agropecuarias, deforestación, construcción de viviendas y las actividades petroleras, descritas en 18 de los 35 sitios de muestreo (Tabla 1), lo que ha disminuido la cobertura vegetal afectando el microhábitat y hábitat de las especies residentes y migratorias (Barba-Macías et al. 2015, De la Rosa-Velázquez et al. 2017). Así como a la presencia de especies exóticas y la sobreexplotación del recurso (Wakida-Kusunoki et al. 2007, Sánchez et al. 2015b, Mendoza-Carranza et al. 2018). Sumado a lo anterior, una mayor presencia de especies de afinidad estuarino marina podría estar incidiendo de alguna manera en la competencia por los recursos y una mejor adaptación a los hábitats que han sido alterados (Lee y Bell 1999).

La diversidad de peces dulceacuícolas reportada (33 spp.) es mayor a las $28 \mathrm{spp}$. registradas por Macossay-Cortez et al. (2011). Sin embargo, se observa una reducción en la ocurrencia de las especies en los ambientes lacustres. Ellos mencionaron 15 especies nativas (siete especies de cíclidos, tres de pecílidos, dos de carácidos, dos eleótridos y Ophisternon aenigmaticum Rosen y Greenwood) registradas en más del $40 \%$ de las lagunas (Tabla 4). En comparación, en este estudio sólo nueve especies nativas ( $A$. finitimus, $C$. aguadulce, $D$. anale, D. petenense, T. helleri, T. meeki, T. pasio- nis, T. salvini, V. melanurus) fueron frecuentemente recolectadas. En contraste, la captura de las nueve especies de pecílidos ha disminuido ya que solo se registraron en 21 de 39 localidades de muestreo y debido a la talla de estos peces vivíparos, menor de $50 \mathrm{~mm}$ de LP, pueden ser indicadores de degradación de hábitat como lo mencionan Olden et al. (2007) y Bennet y Conway (2010) para los peces de talla pequeña. La dominancia espacial del bagre armado no nativo-invasor presente en 89 y $40 \%$ de las lagunas y ríos, respectivamente, puede indicar la relación entre perturbación y sucesión (Sánchez et al. 2019) (Tabla 4). Esta tendencia a la disminución de los peces dulceacuícolas ha recibido poca atención (Olden et al. 2007, Villéger et al. 2015), ya que los pocos registros de algunas especies y la falta de información sobre su biología son temas pendientes que deben ser abordados para la conservación de las especies. En este estudio se observaron a 15 dulceacuícolas (de las 33 spp. reportadas) con ocurrencia baja (menos de cuatro registros). Resaltan los casos de R. guatemalensis y Atherinella alvarezi (Díaz-Pardo) con un solo registro, la primera incluida en la NOM-059 bajo protección especial, y la segunda reportada con mayor frecuencia por Macossay-Cortez et al. (2011) hasta en una tercera parte de sus sitios de recolecta e incluida en la Lista Roja de la IUCN.

\section{CONCLUSIONES}

El incremento del $25 \%$ registrado en la riqueza íctica coincidió con la hipótesis planteada en dos sentidos. Primero asociado con el incremento de la diversidad por la mayor representatividad de peces marino-estuarinos, y la dominancia de peces migratorios diádromos. La limitación referente al desconocimiento de la diversidad íctica en la Reserva se puede explicar por los restringidos monitoreos exhaustivos con dispositivos de muestreo que cubra tanto la selectividad de la captura necesaria como los diferentes hábitats y escalas temporales. El segundo, vinculado a la ausencia de siete especies dulceacuícolas y la disminución en la frecuencia de las mismas en un humedal que recibe la descarga del río más caudaloso en el sureste del Golfo de México. 
Las causas de esta disminución puede explicarse por los esperados efectos negativos de las perturbaciones ambientales, entre las que sobresalen la disminución de hábitat que favorecen la diversidad íctica, la sobrepesca, y el incremento de la abundancia de especies invasoras no nativas. En contraste, la dominancia de peces migratorios diádromos y la presencia frecuente de algunos potamodromos (ambos en $79 \%$ de las localidades) indica la conservación de la interconexión hidráulica entre sistemas ríos y lagunas. Los resultados también indican la necesidad de valorar la condición de algunos peces dulceacuícolas como posibles especies migratorias sin delimitar per se a los peces que realizan grandes desplazamientos y tomar como una efectiva migración (incluso si se realiza de manera parcial solo por algunos individuos de una población) los movimientos laterales que algunas especies presentan durante su ontogenia para satisfacer sus necesidades de sobrevivencia. En este sentido, tratar de cubrir los vacíos de información biológica de algunas especies podría aclarar su condición de potamodromos. Se presenta un panorama más preciso de la riqueza de los peces de la RBPC que ahora alberga el $37 \%$ de la diversidad de especies registradas en las cuencas de los ríos Grijalva-Usumacinta.

\section{AGRADECIMIENTOS}

Al proyecto LH002 SIB-CONABIO, Alberto Macossay y Henrry Reyes por su apoyo durante las recolectas y el procesamiento de los registros.

\section{LITERATURA CITADA}

Adams AJ, Hill JE, Samoray C (2011) Characteristics of spawning ground fidelity by a diadromous fish: a multiyear perspective. Environmental Biology of Fishes 92: 403-411.

Álvarez-Pliego N, Sánchez AJ, Florido R, Salcedo MA (2015) First record of South American suckermouth armored catfishes (Loricariidae, Pterygoplichthys spp.) in the Chumpan River system, southeast Mexico. Biolnvasion Records 4: 309-314.

Álvarez-Pliego N, Sánchez AJ, Florido R, Salcedo MA, Macossay-Cortez A, Brito R, Reyes, H (2016) New records and extension of geographical distribution of Heterophallus echeagarayi (Poeciliidae) in the Usumacinta Province, Mexico. Cybium 40: 178-180.

Arévalo-Frías W, Mendoza-Carranza M (2012) Larvas y juveniles de peces de la Reserva de la Biosfera Pantanos de Centla y sus zona costera adyacente. En: Sánchez AJ, Chiappa-Carrara X, Brito-Pérez R (eds.). Recursos acuáticos costeros del sureste. Vol. 2 Mérida, México. pp: 242-269.

Armbruster JW, Page LM (2006) Redescription of Pterygoplichthys punctatus and description of a new species of Pterygoplichthys (Siluriformes: Loricariidae). Neotropical Ichthyology 4: 401-409.

Barba-Macías E, Valadez-Cruz F, Pinkus M, Pinkus M, Juárez Flores J (2015) Reserva de la Biosfera de Pantanos de Centla: Aspectos Socio-Ambientales Prioritarios. En: Ortega-Rubio A, Pinkus-Rendón MJ, EspitaMoreno IC (eds.). Las Áreas Naturales Protegidas y la Investigación Científica en México. Centro de Investigaciones Biológicas del Noroeste / Universidad Autónoma de Yucatán / Universidad Michoacana de San Nicolás de Hidalgo. México. pp: 1-15.

Bennett MG, Conway KW (2010) An overview of North America's diminutive freshwater fish fauna. Ichthyological Exploration of Freshwaters 21: 63-72.

Benitez JP, Dierckx A, Matondoa BN, Rollinb X, Ovidio M (2018) Movement behaviours of potamodromous fish within a large anthropized river after the reestablishment of the longitudinal connectivity. Fisheries Research 207: 140-149. 
Betancur-R. R, Broughton RE, Wiley EO, Carpenter K, López JA, Li C, et al. (2013) The tree of life and a new classification of bony fishes. PLOS Currents Tree of Life 1-41.

Calzada-Ruíz D, Álvarez-González CA, Peña E, Jiménez-Martínez LD, Alcantar-Vázquez JP, Becerril-Morales F, Martínez-García R, Camarillo-Coop S (2019) Lipid requirement using different oil sources in Mayan cichlid Cichlasoma urophthalmus larvae (Percoidei: Cichlidae). Latin American Journal of Aquatic Research 47: 331-341.

Castillo-Domínguez A, Melgar-Valdes CE, Barba E., Rodiles-Hernández R, Navarrete AJ, Perera-García MA, Cuenca-Soria CA, Hernández-Gómez RE (2015) Composición y diversidad de peces del río San Pedro, Balancán, Tabasco, México. Hidrobiológica 25: 285-292.

Castillo-Torres PA, Martínez-Meyer E, Córdova-Tapia F, Zambrano L (2017) Potential distribution of native freshwater fish in Tabasco, Mexico. Revista Mexicana de Biodiversidad 88: 415-424.

Castro-Aguirre JL, Espinosa Pérez H, Schmitter-Soto J (1999) Ictiofauna estuarino-lagunar y vicaria de México. Mexico City: Noriega-Limusa, IPN. 711 pp.

Cazzanelli M, Soria-Barreto M, Castillo MM, Rodiles-Hernández R (2021) Seasonal variations in food web dynamics of floodplain lakes with contrasting hydrological connectivity in the Southern Gulf of Mexico. Hydrobiologia 848: 773-797.

CONANP (2018) Región Planicie Costera y Golfo de México. https://www.gob.mx/conanp/documentos/regionplanicie-costera-y-golfo-de-mexico?state=published. Fecha de consulta: 16 de junio de 2020.

Cruz-Ramírez AK, Salcedo MA, Sánchez AJ, Barba-Macias E, Mendoza-Palacios JD (2019) Relationship among physicochemical conditions, chlorophyll-a concentration, and water level in a tropical river-floodplain system. International Journal of Environmental Science and Technology 16: 3869-3876.

Chapman BB, Skov C, Hulthén K, Brodersen J, Nilsson PA, Hansson LA, Brönmark C (2012) Partial migration in fishes: definitions, methodologies and taxonomic distribution. Journal of Fish Biology 81: 479-499.

De la Rosa-Velázquez MI, Espinoza-Tenorio A, Díaz-Perera MA, Ortega-Argueta A, Ramos-Reyes R, Espejel I (2017) Development stressors are stronger than protected area management: A case of the Pantanos de Centla Biosphere Reserve, Mexico. Land Use Policy 67: 340-351.

Edge CB, Fortin MJ, Jackson DA, Lawrie D, Stanfield L. Shrestha N (2017) Habitat alteration and habitat fragmentation differentially affect beta diversity of stream fish communities. Landscape Ecology 32: 647-662.

Fricke R, Eschmeyer WN, Van der Laan R (2020) Eschmeyer's catalog of fishes: Genera, species, references. http://researcharchive.calacademy.org/research/ichthyology/catalog/fishcatmain.asp. Fecha de consulta: 17 junio de 2020.

Froese R, Pauly D (2021) FishBase.World Wide Web electronic publication. www.fishbase.org. Fecha de consulta: 17 febrero de 2021.

Galván-Quesada S, Doadrio I, Alda F, Perdices A, Reina RG, García Varela M, et al. (2016) Molecular Phylogeny and Biogeography of the Amphidromous Fish Genus Dormitator Gill 1861 (Teleostei: Eleotridae). PLoS ONE 11(4): e0153538. DOI: 10.1371/journal.pone.0153538

Girard MG, Davis MP, Smith WL (2020) The Phylogeny of Carangiform Fishes: Morphological and Genomic Investigations of a New Fish Clade. Copeia 108: 265-298.

Gómez-González AD, Velázquez-Velázquez E, Anzueto-Calvo MJ, Maza-Cruz MF (2015) Fishes of the Grijalva River basin of Mexico and Guatemala. Check List 11: 1726. 
Granados-Dieseldorff P, Christensen MF, Kihn-Pineda PH (2012) Fishes from Lachuá Lake, Upper Usumacinta Basin, Guatemala. Check List 8: 95-101.

Grenz C, Fichez R, Álvarez-Silva C, Calva-Benítez L, Conan P, Contreras-Ruiz Esparza A, Denis L, Díaz-Ruiz S, Douillet P, Gallegos-Martínez ME, Ghiglione JF, Gutiérrez-Mendieta FJ, Origel-Moreno M, Márquez-García AZ, Muñoz-Caravaca A, Pujo-Pay M, Torres-Alvarado T, Zavala-Hidalgo J (2017) Benthic ecology of tropical coastal lagoons: Environmental changes over the last decades in the Términos Lagoon, Mexico C. R. Geoscience 349: 319-329.

Guadarrama MA, Ortiz G (2000) Análisis de la flora de la Reserva de la Biosfera Pantanos de Centla, Tabasco. Universidad y Ciencia 15: 67-104.

INE (2000) Programa de manejo Reserva de la Biósfera Pantanos de Centla, México. Instituto Nacional de Ecología. Ciudad de México. 222p.

Lee CE, Bell MA (1999) Causes and consequences of recent freshwater invasions by saltwater animals. Trends Ecology and Evolution 14: 284-288.

Liu X, Wang H (2018) Effects of loss of lateral hydrological connectivity on fish functional diversity. Conservation Biology 32: 1336-1345.

Lucas MC, Baras E (2001) Migration of freshwater fishes. Blackwell Science. Oxford. 420p.

Macossay-Cortez A, Sánchez AJ, Florido R, Huidobro L, Montalvo-Urgel H (2011) Historical and environmental distribution of ichthyofauna in the tropical wetland of Pantanos de Centla, southern Gulf of Mexico. Acta Ichthyologica et Piscatoria 41: 229-245.

McBride RS, Matheson RE (2011) Florida's diadromous fishes: biology, ecology, management, and conservation. Florida Scientist 74: 187-213.

McCormick SD, Farrell AP, Brauner CJ (2013) Fish physiology: Euryhaline fishes. Academic Press. London. 559 p.

McMahan CD, Murray CM, Geheber AD, Boeckman CD, Piller KR (2011) Paraneetroplus synspilus is a Junior Synonym of Paraneetroplus melanurus (Teleostei: Cichlidae). Zootaxa 2833: 1-14.

Mendoza-Carranza M, Hoeinghaus DJ, Garcia AM, Romero-Rodriguez A (2010) Aquatic food webs in mangrove and seagrass habitats of Centla Wetland, a Biosphere Reserve in Southeastern Mexico. Neotropical Ichthyology 8: 171-178.

Mendoza-Carranza M, Arévalo-Frías W, Espinoza-Tenorio A, Hernández-Lazo CC, Álvarez-Merino AM, RodilesHernández R (2018) La importancia y diversidad de los recursos pesqueros del río Usumacinta, México. Revista Mexicana de Biodiversidad 89: S131-S146.

Miller RR, Minckley WL, Norris ST (2005) Freshwater fishes of Mexico. The University of Chicago Press. Chicago, USA. 490p.

Milton DA (2009) Living in two worlds: Diadromous fishes, and factors affecting population connectivity between tropical rivers and voasts. In: Nagelkerken I (ed.) Ecological Connectivity among Tropical Coastal Ecosystems. Springer. Dordrecht. Netherlands. pp: 325-355

Minckley WL, Miller RR, Barbourd CD, Schmitter-Soto JJ, Norris SM (2005) Historical ichthyogeography. En: Miller RR, Minckley WL, Norris ST (eds) Freshwater fishes of Mexico. Chicago Univ. Press. USA. pp: 24-47

Montalvo-Urgel H, Sánchez AJ, Florido R, Macossay-Cortez A (2010) Lista de crustáceos distribuidos en troncos hundidos en el humedal tropical Pantanos de Centla, al sur del golfo de México. Revista Mexicana de Biodiversidad 81: S121-S131. 
Morgenstern R (2018) Fishes collected by Emanuel Ritter von Friedrichsthal in Central America between 18381841. Vertebrate Zoology 68: 253-267.

Myers GS (1949) Usage of anadromous, catadromous and allied terms for migratory fishes. Copeia 1949: 89-97.

Myers N, Mittermeier RA, Mittermeier CG, da Fonseca GAB, Kent J (2000) Biodiversity hotspots for conservation priorities. Nature 403: 853-858.

Nelson JS, Grande TC, Wilson MVH (2016). Fishes of the world. Hoboken. John Wiley and Sons. New Jersey. $707 p$.

Nico LG, Butt PL, Johnston GR, Jelks HL, Kail M, Walsh SJ (2012) Discovery of South American suckermouth armored catfishes (Loricariidae, Pterygoplichthys spp.) in the Santa Fe River drainage, Suwannee River basin, USA. Biolnvasions Records 1: 179-200.

Olden JD, Hogan ZS, Vander-Zanden MJ (2007) Small fish, big fish, red fish, blue fish: size-biased extinction risk of the world's freshwater and marine fishes. Global Ecology and Biogeography 16: 694-701.

Palomeque de la CMA, Galindo A, Sánchez AJ, Escalona MJ (2017) Pérdida de humedales y vegetación por urbanización en la cuenca del río Grijalva, México. Investigaciones Geográficas 68: 151-172.

Pease AA, Mendoza-Carranza M, Winemiller KO (2018) Feeding ecology and ecomorphology of cichlid assemblages in a large Mesoamerican river delta. Environmental Biology of Fishes 101: 867-879.

Puc-Carrasco G, Olivera-Gómez LD, Arriaga-Hernández S, Jiménez-Dominguez D (2016) Relative abundance of antillean manatees in the Pantanos de Centla Biosphere Reserve in the coastal plain of Tabasco, México. Ciencias Marinas 42: 261-270.

Reséndez-Medina A, Salvadores ML (2000) Peces de la Reserva de la Biósfera Pantanos de Centla. Resultados preliminares. Universidad y Ciencia 15: 141-146.

Reyes-Ramírez H, Florido R, Álvarez-Pliego N, Sánchez AJ, Salcedo MA (2017) Hábitos alimenticios de Cathorops aguadulce (Siluriformes: Ariidae) en un ecosistema estuarino al sur del golfo de México. Hidrobiológica 27: $163-173$.

Reyes-Rámirez H, Álvarez-Pliego N, Sánchez AJ, Espinosa-Pérez H, Florido R, Salcedo MA (2018) Registros limnéticos de Hypanus sabinus (Myliobatiformes: Dasyatidae) en la cuenca del río Grijalva, sur del Golfo de México. Revista de Biología Marina y Oceanografía 53: 141-145.

Riede K (2004) Global register of migratory species - from global to regional scales. Final Report of the R\&DProjekt 80805 081. Federal Agency for Nature Conservation. Bonn, Germany. 329p.

Salcedo MA, Sánchez AJ, De la Lanza G, Kampichler C, Florido R (2012) Condición ecológica del humedal tropical Pantanos de Centla. En: Sánchez AJ, Chiappa-Carrara X, Brito-Pérez R (eds.) Recursos acuáticos costeros del sureste. Vol. 2. CONCYTEY. Mérida, México. pp: 112-136

Sánchez AJ, Florido R, Macossay-Cortez A, Cruz-Ascencio M, Montalvo-Urgel H, Garrido-Mora A (2012a) Distribución de macroinvertebrados acuáticos y peces en cuatro hábitats en Pantanos de Centla, sur del golfo de México. En: Sánchez AJ, Chiappa-Carrara X, Brito-Pérez R (eds.) Recursos acuáticos costeros del sureste. Vol. 2. CONCYTE.Y Mérida, México. pp: 416-443.

Sánchez AJ, Florido R, Salcedo MA, Ruiz-Carrera V, Montalvo-Urgel H, Raz-Guzman A (2012b) Macrofaunistic diversity in Vallisneria americana Michx. in a tropical wetland, Southern Gulf of Mexico In: Mahamane A (ed.) Diversity of ecosystems. InTech. United Kingdom. Pp: 1-26 
Sánchez AJ, Salcedo MA, Florido R, Mendoza JD, Ruiz-Carrera V, Álvarez-Pliego N (2015a) Ciclos de inundación y conservación de servicios ambientales en la cuenca baja de los ríos Grijalva-Usumacinta. ContactoS 97: 5-14.

Sánchez AJ, Florido R, Álvarez-Pliego N, Salcedo MA (2015b) Distribución de Pterygoplichthys spp. (Siluriformes: Loricariidae) en la cuenca baja de los ríos Grijalva-Usumacinta. Revista Mexicana de Biodiversidad 86: 1099-1102.

Sánchez AJ, Álvarez-Pliego N, Espinosa-Pérez H, Florido R, Macossay-Cortez A, Barba E, Salcedo MA, GarridoMora A. (2019) Species richness of urban and rural fish assemblages in the Grijalva Basin floodplain, southern Gulf of Mexico. Cybium 43: 239-254.

Schmitter-Soto JJ (2007) A systematic revision of the genus Archocentrus (Perciformes: Cichlidae), with the description of two new genera and six new species. Zootaxa 1603: 1-76.

Schmitter-Soto JJ (2017) A revision of Astyanax (Characiformes: Characidae) in Central and North America, with the description of nine new species. Journal of Natural History 51: 1331-1424.

SEMARNAT (2010) Norma Oficial Mexicana. NOM-059-SEMARNAT-2010, Protección ambiental-Especies nativas de México de flora y fauna silvestres-Categorías de riesgo y especificaciones para su inclusión, exclusión o cambio-Lista de especies en riesgo. Diario Oficial de la Federación, 30 de diciembre. 78p.

Soria-Barreto M, González-Díaz AA, Castillo-Domínguez A, Álvarez-Pliego N, Rodiles-Hernández R (2018) Diversidad íctica en la cuenca del Usumacinta, México. Revista Mexicana de Biodiversidad 89: S100-S117.

Schultz ET, McCormick SD (2012) Euryhalinity in an evolutionary context. Euryhaline Fishes. EEB Articles 2013: 477-533.

Toledo-Solis F, Martinez-Garcia R, Galaviz M, Hilerio-Ruiz A, Alvarez-González CA, Rodrigáñez M (2020) Protein and lipid requirements of three-spot cichlid Cichlasoma trimaculatum larvae. Fish Physiology and Biochemistry 46 23-37.

Torres-Martínez A, Sánchez AJ, Álvarez-Pliego N, Hernandez-Frannyutti A, López-Hérnandez JC, Bautista-Regil J (2017) Gonadal histopathology of fish from La Pólvora urban lagoon in the Grijalva basin. Revista Internacional de Contaminación Ambiental 33: 713-717.

Trewavas E (1983) Tilapiine Species of the Genera Sarotherodon, Oreochromis and Danakilia. London: British Museum Natural History 583p.

UICN (2020) Lista Roja de Especies Amenazadas de la UICN. Versión 2020-2. https://www.iucnredlist.org. Fecha de consulta: 09 de julio de 2020.

Velázquez-Aguirre L, Ordaz-Ayala A (1992) Provincia hidrogeológica de México. Boletín de la Sociedad Geológica Mexicana 52: 15-33.

Villéger S, Blanchet S, Beauchard O, Oberdorff T, Brosse S (2015) From current distinctiveness to future homogenization of the world's freshwater fish faunas. Diversity and Distributions 21: 223-235.

Wakida-Kusunoki AT, Ruiz-Carus R, Amador-Del Ángel LA (2007) Amazon sailfin catfish, Pterygoplichthys pardalis (Castelnau, 1855) (Loricariidae), another exotic species established in Southeastern Mexico. The Southwestern Naturalist 52: 141-144.

Wu L-W, Liu C-C, Lin S-M (2011) Identification of exotic sailfin catfish species (Pterygoplichthys, Loricariidae) in Taiwan based on morphology and mtDNA sequences. Zoological Studies 50: 235-246. 
Zhang Q, Dong X, Chen Y, Yang X, Xu M, Davidson TA, Jeppesen E (2018) Hydrological alterations as the major driver on environmental change in a floodplain Lake Poyang (China): Evidence from monitoring and sediment records. Journal of Great Lakes Research 44: 377-387.

Zydlewski J, Wilkie MP (2012) Freshwater to Seawater Transitions in Migratory Fishes. Euryhaline Fishes, 253326. 